\title{
Stochastic resonance in colloidal systems
}

\author{
D. Babič ${ }^{1,2}$, C. Schmitt ${ }^{1}$, I. Poberaj ${ }^{2}$ and C. Bechinger ${ }^{1}$ \\ 1 2. Physikalisches Institut, Universität Stuttgart \\ Pfaffenwaldring 57, 70550 Stuttgart, Germany \\ 2 Faculty of Mathematics and Physics, University of Ljubljana \\ Jadranska 19, 1000 Ljubljana, Slovenia
}

\author{
PACS. 02.50.Ey - Stochastic processes. \\ PACS. 05.40.Jc - Brownian motion. \\ PACS. 82.70.Dd - Colloids.
}

\begin{abstract}
We investigate the dynamical properties of a colloidal particle in a double-well potential which is periodically modulated in time. In case of the modulation corresponding to a tilt of the potential (asymmetric modulation), stochastic resonance is observed when the modulation frequency $\nu_{\Omega}$ matches one half of the Kramers frequency of the unperturbed potential. In contrast, when only the potential barrier height is modulated (symmetric modulation), no synchronization between the modulation and the particle dynamics is observed as demonstrated by the lack of a peak in the power spectrum at $\nu_{\Omega}$.
\end{abstract}

The optimal performance of many devices is notoriously deteriorated by the presence of noise. In nonlinear, e.g. bistable systems, however, the presence of noise can improve the detection of weak periodic signals. This intriguing and apparently paradoxical phenomenon which is known as stochastic resonance (SR) has led, even after more than 20 years since its discovery, to a general and prolonged interest (for a review see, e.g., [1]). Although SR as the explanation for the periodic occurrence of ice ages, where the basic concept was originally introduced by Benzi et al. [2], is still under discussion, by now the existence of SR has been unambiguously demonstrated in physical systems such as Schmitt-Triggers [3], ring lasers [4], or vertical cavity surface emitting lasers [5]. In addition there is also an increasing number of biological systems where some of the characteristic features of $\mathrm{SR}-e . g$. , a maximum in the signal-to-noise ratio as a function of the noise amplitude - were detected (for a review see, e.g., [6]).

While in most experimental systems the noise - one of the most important ingredients of SR - is artificially introduced by an external source, only a few cases were studied where it is inherently present. Colloids - mesoscopic particles which are coupled to a thermal bath and therefore undergo Brownian motion - are a good example of a system with intrinsic noise. Because the length and time scales in colloidal systems are conveniently accessible with experiments, they provide excellent model systems for statistical mechanics in general and as such also for SR experiments.

Simon and Libchaber investigated the motion of a colloidal particle in a double-well potential which was periodically modulated with period $T_{\Omega}[7]$. When $T_{\Omega}$ was close to twice 
the Kramers time, thermally driven stochastic hopping of the particle across the barrier was found to be synchronized with the periodic force modulation. This was inferred from the analysis of the residence time distribution $P_{\text {res }}(T)$, showing the probability that a particle stays over the time $T$ within a certain potential well. Their results, however, did not allow for a conclusive interpretation of $\mathrm{SR}$ as a genuine resonant process as was later pointed out by other authors [8]. In addition, it remained unclear why the peak height in $P_{\text {res }}$ increased at longer times since in the case of statistically independent events (such as the hopping of a particle across the barrier) an exponentially decaying envelope of $P_{\text {res }}$ is expected.

In this letter, we study the dynamics of a colloidal particle in a periodically time-modulated double-well potential. A pair of fast optical tweezers, based on an acousto-optic deflection system, is used to create suitable light potentials for a colloidal particle. We investigated two types of potential modulation: i) a generic case of SR with asymmetric modulation inducing a periodic tilting of a double-well potential and therefore breaking its spatial symmetry and ii) a symmetric modulation, where the potential barrier height was periodically modulated and the symmetry of the double-well potential was preserved at all times. While in the first case our measurements clearly demonstrate SR, the latter case shows a significantly different behavior. Although in both cases a redistribution of the hopping events in $P_{\text {res }}$ compared to the unmodulated system is observed, the analysis of the corresponding power spectra shows that no synchronization occurs in the case of symmetric modulation.

The experimental setup was composed of three main parts - a pair of optical tweezers [9], a sample cell with deionizing circuit and a data acquisition system. The optical tweezers were constructed around a custom-built microscope with two coaxially placed, vertically oriented microscope objectives in a confocal configuration and a sample cell containing colloidal particles. The laser beam was focused by the upper objective $(50 \times, N A=0.6)$ which also served as a condenser for the particle illumination. The lower objective $(40 \times, N A=0.5)$, together with a low-pass optical filter blocking the laser light, was used to image the particles onto a CCD camera. As laser source we used the $\mathrm{TEM}_{00}$ output of a frequency-doubled solid-state laser at $532 \mathrm{~nm}$. The beam was first expanded to a diameter of approximately $3.5 \mathrm{~mm}$, guided through a two-axis acousto-optic deflector (AOD) and finally inserted into the entrance aperture of the upper objective by a telecentric lens system. Together with a custom-built driving electronics and control software, the AOD enabled us to create multiple optical traps by time sharing the laser beam with a switching rate of $50 \mathrm{kHz}$ [10]. The range of the optical tweezers in the sample plane was $150 \times 150 \mu \mathrm{m}$, with nanometer resolution and repeatability on the order of $100 \mathrm{~nm}$. The intensity of each trap could be set with 12-bit resolution. Measurements were done with $1.2 \mu \mathrm{m}$ diameter polystyrene particles obtained from IDC. A highly diluted suspension was inserted into a sample cell via a standard deionizing circuit described elsewhere [11]. The experiments were performed at room temperature with stability of about $\pm 1 \mathrm{~K}$.

The particle motion was recorded with a CCD camera which was connected to a PCinstalled frame grabber. Data was acquired at 20 frames per second and recorded in a compressed format onto a hard drive. Proprietary software was used for particle recognition, trajectory reconstruction, analysis of the hopping events and determination of the potential shape created by the optical tweezers.

Double-well potentials were created by placing two optical traps at a distance of approximately $1.6 \mu \mathrm{m}$. Due to time sharing of the laser beam between the two traps, possible interference effects were absent. The switching time between the traps was $20 \mu \mathrm{s}$, so the potential can be regarded as quasistatic. Only lateral confinement of the particle was induced by the gradient light force. Vertically the particle was pushed by the pressure of the laser beam against the lower wall of the sample cell to the distance of approximately $500 \mathrm{~nm}$, where it was equilibrated by the electrostatic repulsion. 


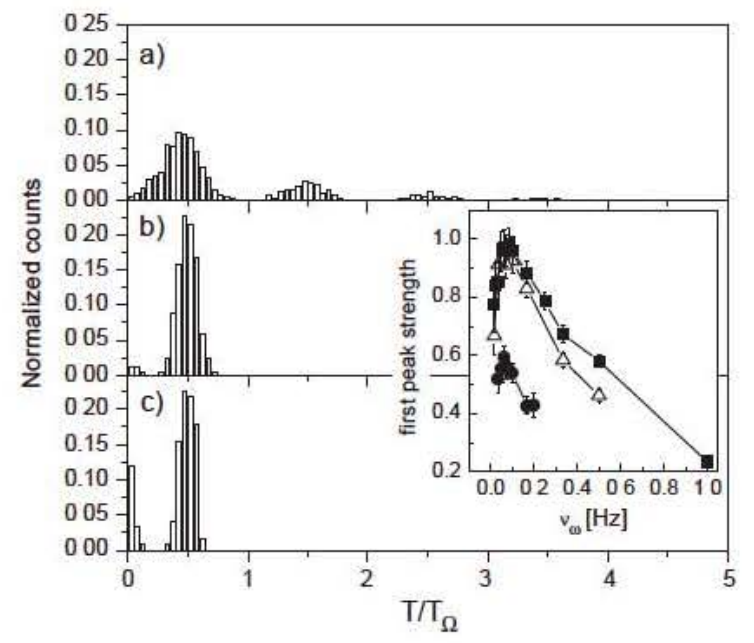

Fig. 1 - Normalized (to the total number of events) residence time distributions as a function of $T / T_{\Omega}$ at a) $T_{\Omega}=2$, b) 14 , and c) $40 \mathrm{~s}$ for the case of asymmetric modulation with an intensity modulation amplitude of $\pm 15 \%$. The inset shows the strength of the first peak vs. modulation frequency $\nu_{\Omega}=1 / T_{\Omega}$ for different modulation amplitudes: (⿴) $\pm 15 \%,(\triangle) \pm 11 \%$ and $(\bullet) \pm 7 \%$ variation of the mean laser intensity.

For the interpretation of the experimental results it is important to consider three time scales. In an unmodulated double-well potential the average time between particle hops across the barrier with height $\Delta V$ is the Kramers time $T_{\mathrm{K}}=T_{\mathrm{R}} \exp [\beta \Delta V] . T_{\mathrm{R}}$ is the relaxation time within a single potential well and $\beta^{-1}$ is the thermal energy of the system. These two time scales are intrinsic to the system. The third time scale is the period $T_{\Omega}$ of the externally applied modulation. In our experiments $T_{\Omega}$ was varied from values being short to values being long compared to $T_{\mathrm{K}}$, but always significantly longer than $T_{\mathrm{R}}$. We will first discuss the results obtained with asymmetric modulation of the double-well potential corresponding to the generic SR case. The central condition of this case is $T_{\Omega} \approx 2 T_{\mathrm{K}}$ when the stochastic time scale $T_{\mathrm{K}}$ matches the deterministic time scale $T_{\Omega} / 2$ and SR can be observed. This condition can be met either by varying the noise intensity (as done in most experiments) or $T_{\Omega}$ as done here. Secondly, we discuss the case of symmetric modulation where the potential barrier is turned on and off. Finally, we will compare the two cases.

Asymmetric modulation of the double-well potential. - From the particle trajectories we first determined the residence time distribution. In the absence of modulation, $P_{\text {res }}(T)$ decays exponentially for times larger than the intrawell relaxation time $T_{\mathrm{R}}$ (being smaller than $0.1 \mathrm{~s}$ as estimated from the decay of the autocorrelation function of the intrawell particle motion) with the decay constant given by $T_{\mathrm{K}}$. For our experimental conditions $T_{\mathrm{K}}$ was measured to be $7.3( \pm 0.4) \mathrm{s}$. In the presence of a periodic modulation, $P_{\text {res }}$ shows significant deviations from an exponential decay, as is exemplarily demonstrated in figs. 1a-c for $T_{\Omega}=2,14,40 \mathrm{~s}$. For better comparison we normalized $P_{\text {res }}$ with respect to the total number of events and plotted it vs. the dimensionless time $T / T_{\Omega}$. For modulation times smaller than $T_{\mathrm{K}}$, the distribution shows a series of peaks centered at odd multiples of $T_{\Omega} / 2$ (fig. 1a). These peaks appear due to the modulation of the transition probabilities between the potential wells. The separation of the peaks becomes obvious when considering that on average a particle "waits" until the opportunity for a hop is largest. If the particle misses this opportunity it is likely to reside 


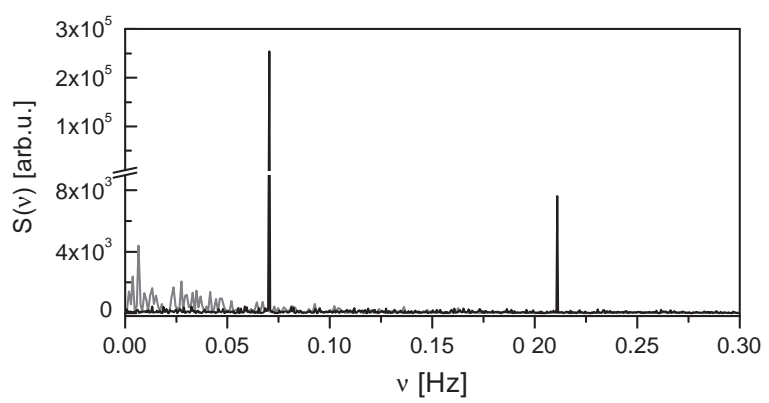

Fig. 2 - Power spectra of particle fluctuations for an unmodulated (gray) and an asymmetrically modulated (black) double-well potential. Modulation corresponds to $\pm 15 \%$ variation of the mean laser intensity. On top of the noise background narrow peaks at the driving frequency $\nu_{\Omega}=0.07 \mathrm{~Hz}$ and its third harmonic are seen.

in the same well for another (or multiple) $T_{\Omega}$. The position of the first peak follows from the fact that there are at most two "good" opportunities per modulation period for the particle to hop. The observed structure of $P_{\text {res }}$ is in very good agreement with numerical calculations [12]. Because thermally activated barrier crossings are statistically independent, the peak height is predicted to decay exponentially [12] which is also in agreement with our experimental findings. This is in contrast to [7], where a resurgence of the peak heights at longer times was observed.

Upon increasing $T_{\Omega}$ towards the resonance condition, the multi-peak structure disappears and the residence time distribution becomes narrower until essentially only a single peak at $T_{\Omega} / 2$ is observed (fig. $1 \mathrm{~b}$ ). In this situation, the stochastic barrier crossing of the particle is maximally synchronized with the periodic modulation. When $T_{\Omega}$ is much higher than $T_{\mathrm{K}}$, the particle hopping becomes less synchronized again which can be seen by the increasing transition probability at short times (fig. 1c).

In order to demonstrate SR in systems where the noise strength remains constant, Gammaitoni et al. suggested to plot the strength (i.e. area) of the $n$-th peak in $P_{\text {res }}$ as a function of $\nu_{\Omega}=1 / T_{\Omega}[8,13,14]$. In case of SR, the curve should exhibit a pronounced maximum and SR can thus be regarded as a bona fide resonance. In the following we applied this criterion to our data although the system is not truly one-dimensional as assumed in [8]. The inset of fig. 1 shows the first-peak strength as a function of $\nu_{\Omega}$ and indeed displays a maximum thus definitely demonstrating SR in a colloidal system. Measurements were performed for three different modulation amplitudes which corresponded to intensity variations of the two laser traps of $\pm 15 \%,(\triangle) \pm 11 \%$ and $(\bullet) \pm 7 \%$, respectively. While the strength of the first peak decreases with smaller modulation amplitude, pronounced maxima are observed in all three cases.

Another way to identify SR relies on the analysis of the power spectrum $S(\omega)$ of the particle fluctuations. Figure 2 shows the results for an unperturbed and a close-to-resonance modulated system (gray and black curves, respectively). The presence of the modulation leads to pronounced peaks at the modulation frequency $\nu_{\Omega}$ and its third harmonic, the latter being in agreement with theoretical predictions $[15,16]$. It is also seen that the black curve always lies below the light curve (apart from the peaks). This is in good agreement with theoretical and numerical studies which demonstrated that the characteristic feature of the $\mathrm{SR}$ is the transmission of power from a broad-band noise spectrum into the peak at $\nu_{\Omega}$ [17]. The absence of peaks at even harmonics of $\nu_{\Omega}$ indicates that our system is well balanced, i.e. any residual tilt of the double-well potential is below our detection limit $[15,16]$. 


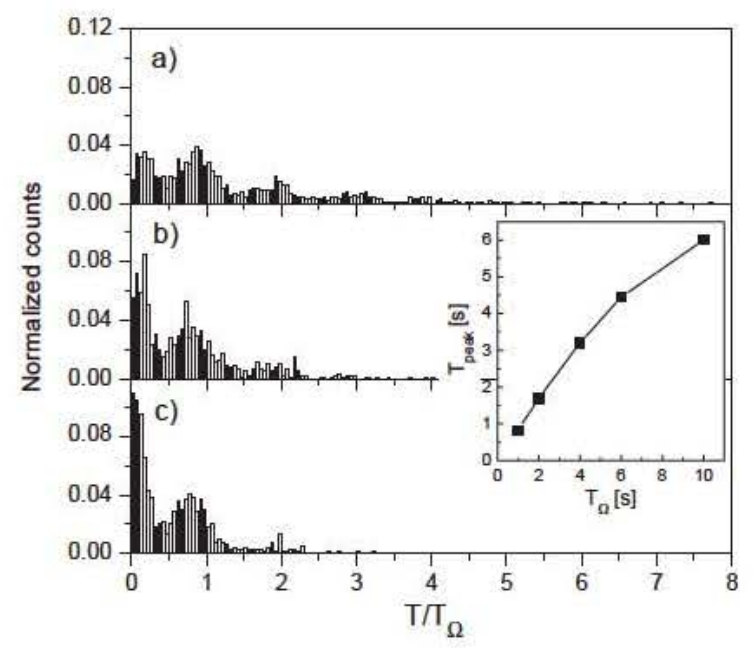

Fig. 3 - Normalized residence time distributions for the case of symmetric modulation at a) $T_{\Omega}=2$, b) 4 and c) $6 \mathrm{~s}$. The inset shows the position of the first peak as a function of $T_{\Omega}$.

Symmetric modulation of the double-well potential. - In addition to the above case which is typically considered in SR, we also studied the situation where the modulation preserves the symmetry of the double-well potential. In this situation the potential is not periodically tilted, instead the potential barrier height is modulated. Experimentally, this was achieved by a third laser trap which was placed in between the other two traps comprising the double-well potential. The intensity of the third beam was modulated in such a way that the potential barrier was periodically turned on and off. Considering first the residence time distribution we note the following distinct features. For $T_{\Omega}<T_{\mathrm{K}}$ (fig. 3a)), $P_{\text {res }}$ shows a similar multi-peak structure as for the asymmetric modulation (cf. fig. 1). However, while the separation between the peaks is $T_{\Omega}$ as before, the first peak is no longer located at $T_{\Omega} / 2$ but shifted approximately to $T_{\Omega}$. This is due to the fact that there is only one "good" opportunity for a particle to hop within $T_{\Omega}$. As can be seen from the inset of fig. 3 , the first peak position does not linearly follow $T_{\Omega}$, instead increasing deviations are observed towards larger modulation times. This behavior, together with the apparent underlying structure of $P_{\text {res }}$ in fig. 3, deserves a closer experimental and theoretical analysis which is, however, beyond the scope of the current work. This is also the reason why we did not further investigate the behavior of the first-peak strength vs. $T / T_{\Omega}$. We do though expect it to have a maximum at a certain modulation frequency.

When calculating the power spectrum for the symmetric modulation, in contrast to the asymmetric case, within our experimental resolution no peak at the modulation frequency is observed (fig. 4). This demonstrates that no synchronization occurs for symmetric modulation of the double-well potential. To understand this, one has to recall why synchronization occurs in case of asymmetric modulation of the double-well potential (note that the term synchronization does not imply instantaneous phase locking or frequency synchronization [18] but rather synchronization in an averaged sense, as indicated by the first peak in $P_{\text {res }}$ [19]). Since the probabilities for a particle to hop in either direction are not identical, the particle motion is in phase with the external modulation. If, e.g., the probability to find the particle at time $t_{0}$ in the left potential well is highest, then the particle is most likely to occupy the same (!) well at later times $t=t_{0}+n T_{\Omega}(n=1,2,3, \ldots)$. In the case of symmetric modulation, however, 


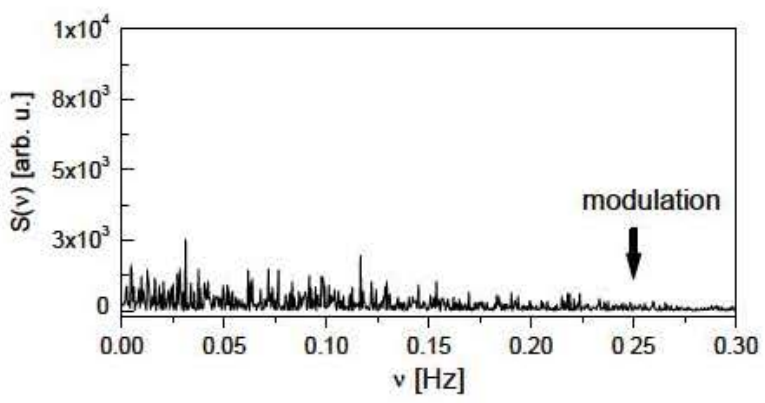

Fig. 4 - Power spectrum for symmetric modulation. No peak at the external modulation frequency $\nu_{\Omega}=0.25 \mathrm{~Hz}$ (arrow) is visible.

the escape probabilities are identical for both wells at all times. Therefore, the probability to find a particle in any of the two wells is constant. As a consequence, the particle position is not connected to the phase of the potential modulation which explains our experimental findings. This qualitative argument is also supported by theoretical results $[16,20]$.

It has been demonstrated in several experiments that there is a close resemblance of the interspike interval histograms in periodically stimulated, e.g. auditory nerve systems to the waiting time distribution observed in SR [1]. To account for the position of the peaks in neuronal experiments at multiples of $T_{\Omega}$, an additional reset mechanism has to be considered which is most likely connected to the repolarization of the neuronal membrane after a depolarizing upstroke of the action potential [21]. Theoretical and numerical results, however, suggest that this is at least not the only possible explanation for the occurrence of peaks at multiples of $T_{\Omega}$ and that a univocal criterion for SR in systems being certainly more complex than a double-well potential should not entirely rely on interval histograms. This is now also supported by our experimental findings.

Finally, we would like to mention that there is considerable ongoing theoretical and numerical effort in describing the properties of coupled bistable systems which is contrasted by only a few recent experimental studies [22]. Colloidal systems might also be used as convenient model systems in this regime because our experimental setup can create up to several tens individually controlled double-well systems. Along with the possibility to vary the particle interactions in colloidal systems, this allows us to study the effects present in a variety of coupled bistable systems.

In summary, we have investigated the dynamical properties of a colloidal particle in a timemodulated bistable system which has been realized by optical tweezers. Under asymmetric modulation conditions we clearly demonstrated the occurrence of SR. We also demonstrated a significant difference in the behavior of the system under conditions of symmetric modulation which to our opinion deserves further theoretical consideration.

We acknowledge helpful discussions with P. REIMANN and technical support from P. LEIDERER. This work is financially supported by the Deutsche Forschungsgemeinschaft $(1788 / 4-1)$. 


\section{REFERENCES}

11] Gammaitoni L., Hänggi P., Jung P. and Marchesoni F., Rev. Mod. Phys., 70 (1998) 223.

[2] Benzi R., Parisi G., Sutera A. and Vulpiani A., Tellus, 34 (1982) 10.

[3] Fauve S. and Heslot F., Phys. Lett. A, 97 (1983) 5.

[4] McNamara B., Wiesenfeld K. and Roy R., Phys. Rev. Lett., 60 (1988) 2626.

[5] Giacomelli G., Marin F. and Rabbiosi I., Phys. Rev. Lett., 82 (1999) 675.

[6] Hänggi P., Chem. Phys. Chem., 3 (2002) 285.

[7] Simon A. and Libchaber A., Phys. Rev. Lett., 68 (1992) 3375.

[8] Gammaitoni L., Marchesoni F. and Santucci S., Phys. Rev. Lett., 74 (1995) 1052.

[9] Ashrin A., Phys. Rev. Lett., 24 (1970) 156.

[10] Visscher K., Gross S. P. and Block S. M., IEEE J. Quantum Electron., 2 (1996) 1066.

[11] Rudhardt D., Bechinger C. and Leiderer P., Phys. Rev. Lett., 81 (1998) 1330.

[12] Zhou T., Moss F. and Jung P., Phys. Rev. A, 42 (1990) 3161.

[13] Choi M. H., Fox R. F. and Jung P., Phys. Rev. E, 57 (1998) 6335.

[14] Marchesoni F., Gammaitoni L., Apostolico F. and Santucci S., Phys. Rev. E, 62 (2000) 146.

[15] Jung P. and HÄngGi P., Europhys. Lett., 8 (1989) 505.

[16] Jung P. and Hänggi P., Phys. Rev. A, 44 (1991) 8032.

[17] McNamara B. and Wiesenfeld K., Phys. Rev. A, 39 (1989) 4854.

[18] Callenbach L., Hänggi P., Linz S. J., Freund J. A. and Schimansky-Geier L., Phys. Rev. E, 65 (2002) 051110.

[19] Neiman A., Silchenko A., Anishchenko V. and Schimansky-Geier L., Phys. Rev. E, 58 (1998) 7118.

[20] Presilla C., Marchesoni F. and Gammaitoni L., Phys. Rev. A, 40 (1989) 2105.

[21] Longtin A., Bulsara A. R. and Moss F., Phys. Rev. Lett., 67 (1991) 656.

[22] Sharpe J. P., Sungar N., Swaney M., Carrigan K. and Wheeler S., Phys. Rev. E, 67 (2003) 056222. 